\title{
Utilisation de microtubes héparinés pour recueillir le sang destiné à l'analyse chimique, en particulier au dosage du potassium ${ }^{1}$ )
}

\author{
Par J. Mrchod, C. Platsoukas et J. Frer \\ Laboratoire Central de l'Hopital Cantonal Lausanne, Suisse
}

(Eingegangen am 27. Mai 1969)

\begin{abstract}
Des microtubes héparinés ont été employés pour recueillir le sang destiné à l'analyse chimique.
L'anticoagulant prévient la microhémolyse qui intervient lors de la coagulation et surtout l'hémolyse fréquemment observéc dans les récipients de plastique.

Le potassium a été dosé en parallèle dans le sérum et le plasma de 36 personnes en bonne santé en vue d'établir les valeurs normales. Le calcul statistique n'a pas révélé de grandes différences entre les moyennes et les écarts-types. Par contre, des différences individuelles ont été constatées entre potassium sérique et plasmatique, inadmissibles dans $6 \%$ des cas normaux ainsi que dans $20 \%$ de 193 cas de la clinique.

En conclusion, nous recommandons l'emploi de microtubes héparinés pour le dosage des électrolytes, ainsi que pour les dosages des enzymes et autres métabolites, à l'exclusion toutefois des protéines et de l'électrophorèse.
\end{abstract}

\section{The use of beparinised micro-sample tubes for the collection of blood for chemical analysis, especially for potassium}

Heparinized micro-tubes were used for collecting blood analysis

The anticoagulant prevents the microhaemolysis which occurs during the coagulation and the haemolysis frequently observed in plastic material.

The potassium was estimated in both the serum and plasma of 36 healthy subjects and the normal values were established. Slight diffetences between the mean values and the standard deviation of both collectives were shown. On the other hand, individual differences between the potassium levels in serum and plasma were inadmissible in $6 \%$ of the normal subjects and in $20 \%$ of 193 patients.

In conclusion the use of heparinized micro-tubes can be recommended not only for the estimation of the electrolytes, but also for some enzymes and other metabolites, with the exception of proteins; the samples cannot be used for electrophoresis.

Heparinisierte Mikro-Röhrchen werden zur Blutentnahme für chemische Analysen benutzt. Das Antikoagulans verhindert die Mikrohämolyse, die während der Gerinnụng eintritt und die Hämolyse, die häufig in Plastikröhrchen beobachtet wird.

Kalium wurde sowohl im Serum als auch Plasma von 36 gesunden Probanden bestimmt. Normalwerte werden angegeben. Es bestehen geringe Differenzen zwischen dem Mittelwert und der Standardabweichung beider Kollektive. Andererseits waren die individuellen Unterschiede der Kaliumwerte in Serum und Plasma bei 6\% der gesunden Probanden und bei 20\% von 193 Patienten unzulässig groß. Schließlich kann die Verwendung von heparinisierten Mikro-Röhrchen nicht nur für Elektrolytbestimmungen, sondern auch für einige Enzyme und andere Stoffwechselprodukte mit Ausnahme der Proteine empfohlen werden. Die Proben können nicht zur Elektrophorese verwendet werden.

L'extension des méthodes microanalytiques en chimie clinique permet de diminuer le volume del'échantillon de sang nécessaire aux examens, ce qui est particulièrement avantageux en pédiatrie. Il s'ensuit que, de plus en plus, l'acheminement du sang au laboratoire se fait dans des microtubes de $\mathrm{SANZ}^{2}$ ) de polyéthylène ou de polypropylène plutôt que dans les macrotubes en verre.

Ces microtubes de $500-600 \mu l$ présentent des avantages importants sur les tubes de verre de $10 \mathrm{~m} l$ utilisés couramment:

- ils ne sont pas cassants

- ils peuvent être insérés dans des entailles faites dans la fiche même de demande d'examens ce qui rend l'étiquetage superflu en clinique

- ils peuvent être distribués à raison d'un ou deux tubes à chaque poste d'analyses ce qui accélère le rythme

1) Le sujet du présent travail a fait l'objet d'une comminication le 18 avril 1969 a Lugano lors de la 13ème Assemblée Générale de la Société Suisse de Chimie Clinique.

2) Fournisseur: Milian Instruments S. A., Bd. Helvétique 26, CH 1207 Genève. des dosages et rend inutile le poste de répartition du sérum.

Ces microtubes présentent cependant des inconvénients inhérents à la nature même de leur matériau: la coagulation s'y fait plus lentement que dans des tubes de verre. Quelquefois, elle ne se produit qu'après centrifugation (ce qui oblige à répéter l'opération) ou même après prélèvement de la prise à analyser. Dans ce cas, - la fibrine obture les tuyaux des appareils d'analyses automatiques tels que l'AutoAnalyzer ou le photomètre à flamme IL à dilueur automatique.

Un autre inconvénient est que le caillot se sépare souvent mal parce qu'il adhère aux parois. Il est nécessaire de l'en décoller à l'aide d'une baguette de bois ou de matière plastique et de centrifuger une deuxième voire une troisième fois. Cela ne se fait pas sans écrasement des érythrocytes et d'autres éléments figurés qui sont inclus dans le caillot. On est donc fréquemment contraint de doser le potassium dans des sérums plus ou moins hémolytiques.

On sait, par ailleurs, que lors de la coagulation, et sans qu'il y ait hémolyse, il se produit la lyse des thrombo- 
cytes ce qui élève faussement le taux de potassium sérique.

Nous avons pensé qu'il était possible d'éviter ces inconvénients en demandant à notre fournisseur de nous livrer des microtubes contenant de l'héparinate de Li. Des essais ont montré que cet anticoagulant n'influence pas le dosage des électrolytes par les méthodes que nous utilisons, à savoir:

Dosage du $\mathrm{Na}$ et $\mathrm{K}$ au moyen du photomètre à flamme IL à étalon interne de $\mathrm{Li}$, dosage du $\mathrm{Ca}$ au moyen du photomètre à flamme Eppendorf.

Les tubes contenant de l'héparinate de $\mathrm{Li}$ sont bleus, ce qui permet de les distinguer des tubes ordinaires.

Puisque l'addition d'anticoagulant diminue les risques d'hémolyse, évite la lyse des thrombocytes et évite aussi les risques d'écrasement des érythrocytes lors du décollement du caillot, on pouvait prévoir que les valeurs normales du potassium plasmatique devaient être sensiblement plus basses que les valeurs normales établies pour le potassium sérique. Nous avons donc jugé nécessaire de déterminer les valeurs normales du $\mathrm{K}$ plasmatique.

Un collectif de sang de 36 personnes en bonne santé nous a été fourni par le Centre de Transfusion Sanguine de la Croix-Rouge Suisse à Lausanne. Les sangs ont été recueillis d'une part dans des microtubes héparinés et d'autre part dans des microtubes ordinaires.

L'analyse statistique des taux de potassium plasmatique et sérique a donné les résultats figurant dans le tableau 1. On constate que les moyennes ne sont en réalité pas très différentes et les valeurs normales assez proches les unes des autres.

Tab. 1

Dosages $\mathrm{K}^{+}$dans 36 sangs normaux

\begin{tabular}{|c|c|c|}
\hline & Plasma & Sérum \\
\hline $\begin{array}{l}\text { moyenne en mVal } / l \\
\text { écart-type }(\sigma) \\
\text { valeurs normales }( \pm 2 \sigma)\end{array}$ & $\begin{array}{c}3,91 \\
0,31 \\
3,29-4,55\end{array}$ & $\begin{array}{c}4,12 \\
0,37 \\
3,39-4,87\end{array}$ \\
\hline
\end{tabular}

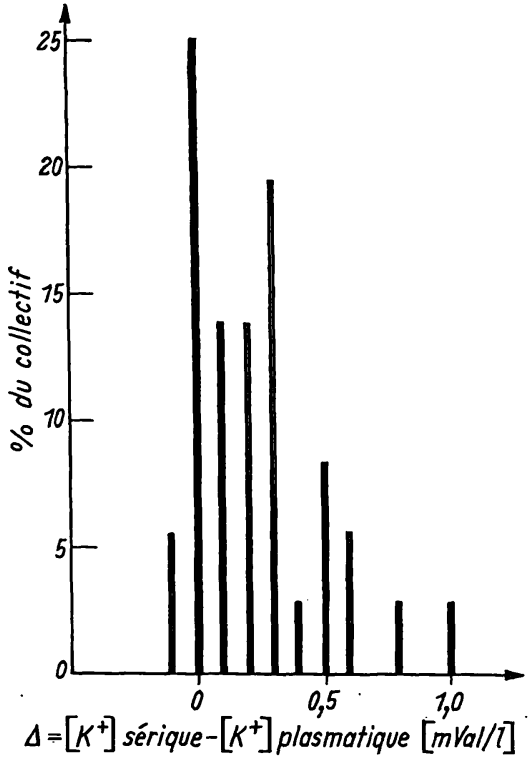

Fig. 1

Répartition des différences entre taux de potassium sérique et plasmatique de 36 cas normaux

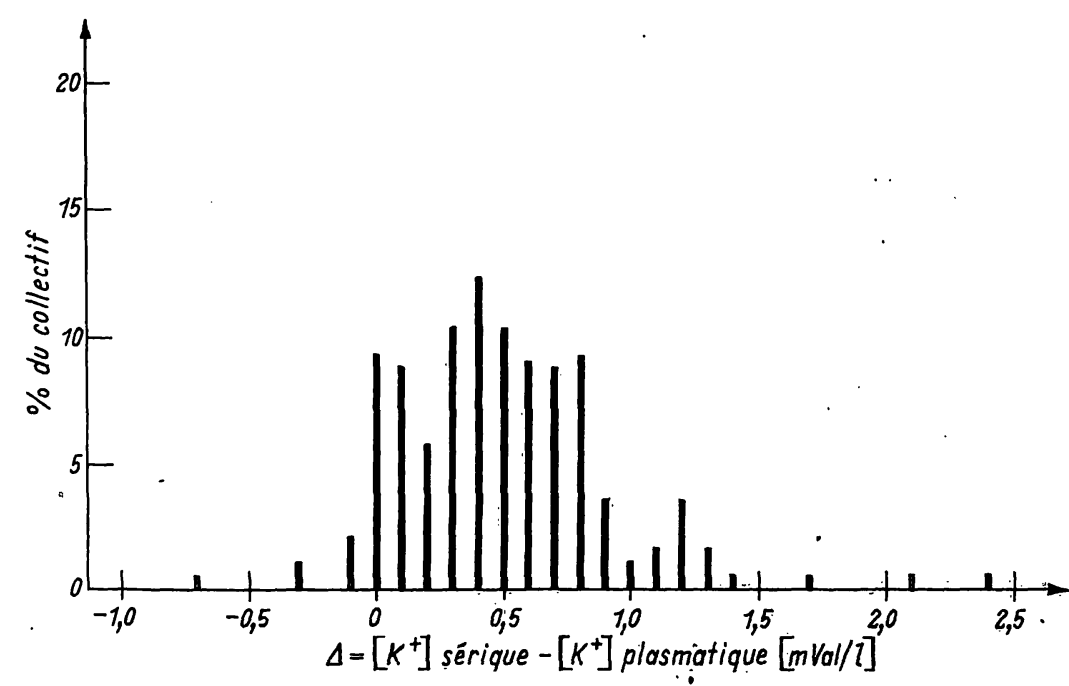

Fig. 2

Répartition des différences entre taux de potassium sérique et plasmatique de 193 cas de la routine 
SCHOTT

$\mathrm{T} 40 \mathrm{~B}$

JENAER GLASWERK SCHOTT \& GEN., MAINZ AQUA PRO INJECTIONE

Wir liefern Destillierautomaten mit Leistungen bis $500 \mathrm{l} / \mathrm{h}$. Die hervorragenden Eigenschaften des Spezialglases DURAN $50{ }^{\circledR}$ und die ausgereifte Konstruktion der Anlagen garantieren Wasserqualitäten, die den Forderungen der internationalen Pharmakopöen entsprechen. Deshalb werden vorzugsweise unsere Anlagen in der pharmazeutischen Industrie und in Krankenhäusern sowie Blutspendezentralen eingesetzt . . .

\section{... z. B. in der Blutspendezentrale}

\section{Bad Kreuznach}

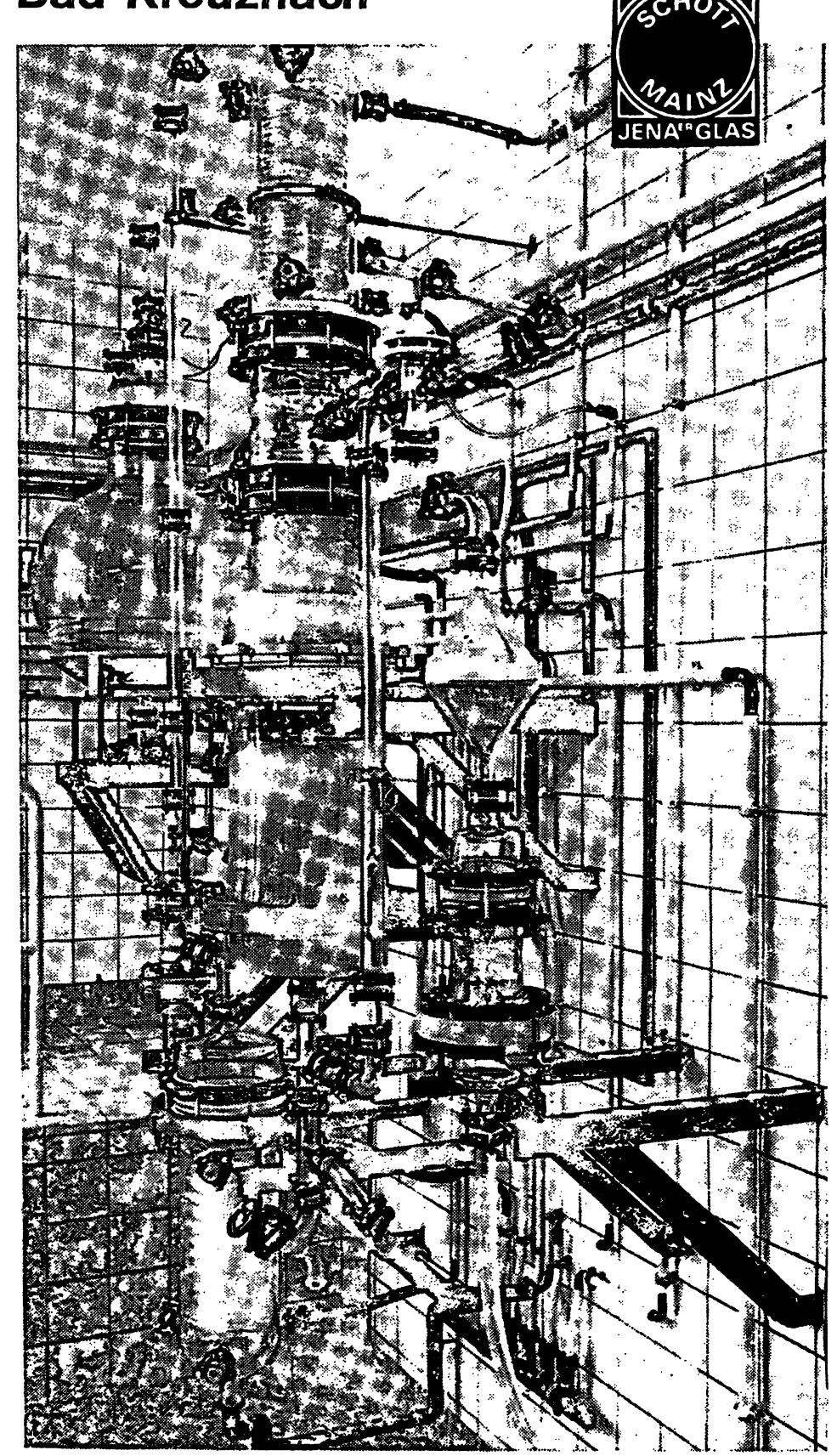

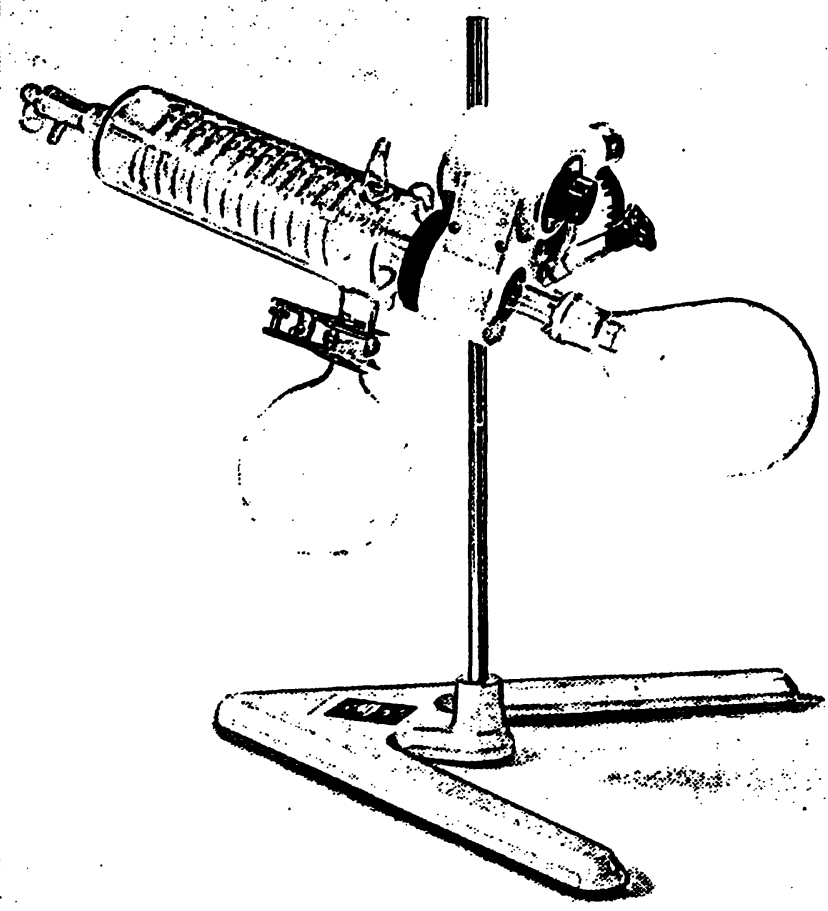

Büchi-Rotavapor R/A

Der bewährte und erfolgreiche Vakuum-Rotationsverdampfer für schonende und rasche Destillation. Optimale Arbeitsbedingungen durch stufenlose Regulierung der Tourenzahl. Nahezu geräuschloser Betrieb, verstellbare Neigung, Auswechselbarkeit aller Teile.

Alleinvertretung für Deutschland:

Colora Meßtechnik GmbH

7073 Lorch/Württ. Postfach 5

T (07172) 6041 , FS 07-248 886

Technische Büros (Verkauf und Kundendienst) : 1000 Berlin 30, Kurfürstenstraße 84, T 135200 2000 Hamburg 19, Osterstraße 63, T 400606

4000 Düsseldorf, Kronprinzenstr. 62, T 178 60, FS 08-587253 6000 Frankfurt (Main), Liebigstr. 24, T 729613 , FS 04-11216 8000 München 2, Dachauer Straße 175, T 5169858

\section{BOOHE}




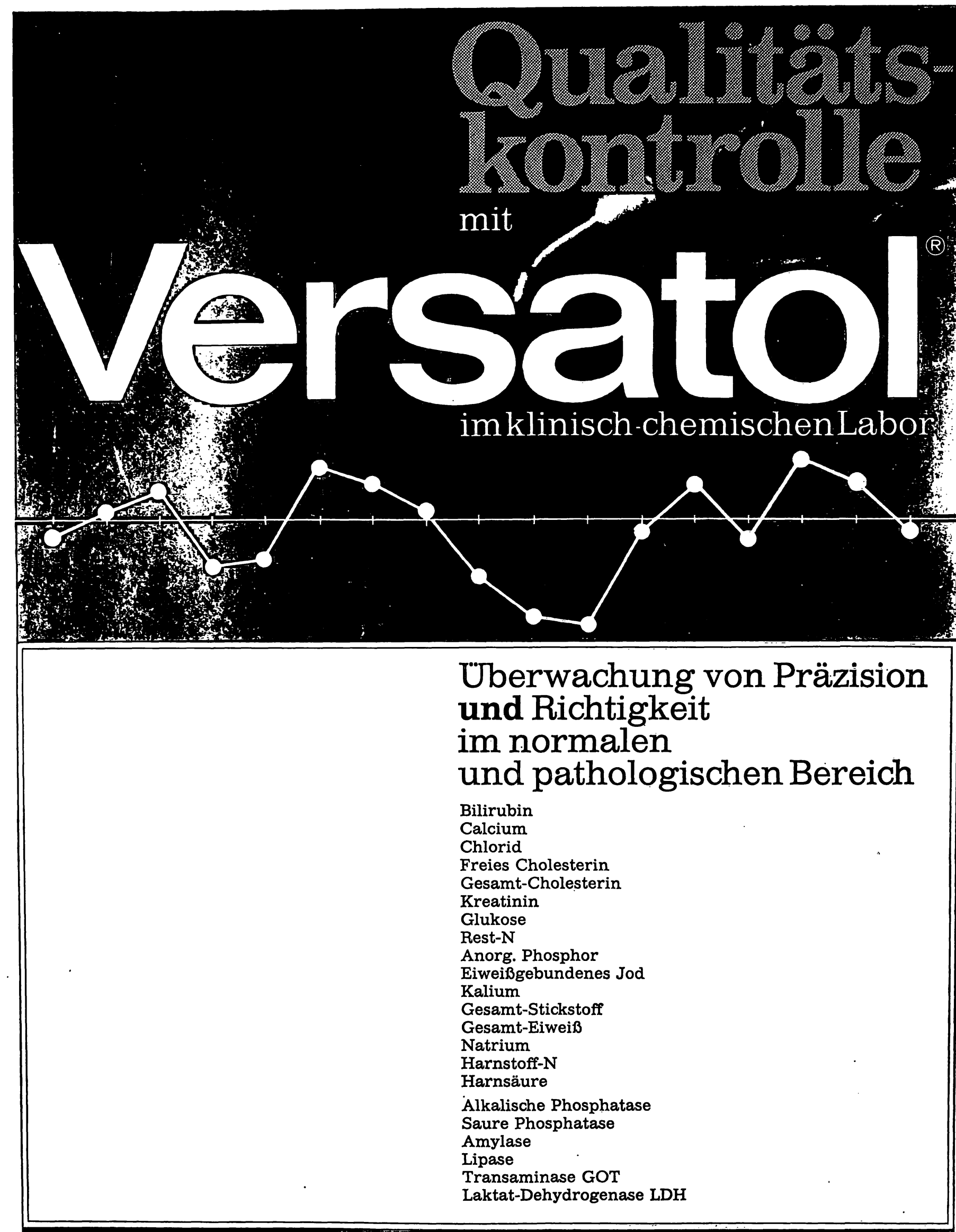

\section{GODECKE}


En raison des conditions de travail qui sont celles d'un laboratoire d'urgence, on pouvait également prévoir que la proportion des taux de $\mathrm{K}$ sériques s'écartant d'une façon inacceptable des taux de $\mathrm{K}$ plasmatiques serait plus forte que pour les cas normaux.

La figure 2 confirme ce pronostic.

Dans $26 \%$ des cas seulement les taux de $\mathrm{K}$ plasmatique et sérique ne diffèrent que de $\pm 0,2 \mathrm{mVal} / l .20 \%$ au lieu de $6 \%$ des valeurs sériques doivent être considérées comme inacceptables.

Il faut toutefois noter qu'il s'agissait là de sérums fortement hémolytiques et que les résultats du dosage étaient en ce cas accompagnés de la remarque «sérum hémo- lytique", cela pour permettre au médecin d'interpréter le résultat et de refaire la prise de sang s'il le jugeait nécessaire.

En conclusion, nous recommandons la généralisation de l'emploi des microtubes héparinés pour recueillir le sang, non seulement en vue du dosage du potassium, mais pour tous les autres dosages, ceux des enzymes y compris, à l'exception de celui des protéines totales et hormis pour l'électrophorèse.

Nous remercions le Centre de Transfusion de la Croix-Rouge Suisse, Section de Lausanne, qui nous a fourni les sangs de personnes en bonne santé.
Dr. J. Frei

Hôpital cantonal

Laboratoire Central

$\mathrm{CH}-1000$ Lausanne/Suisse 